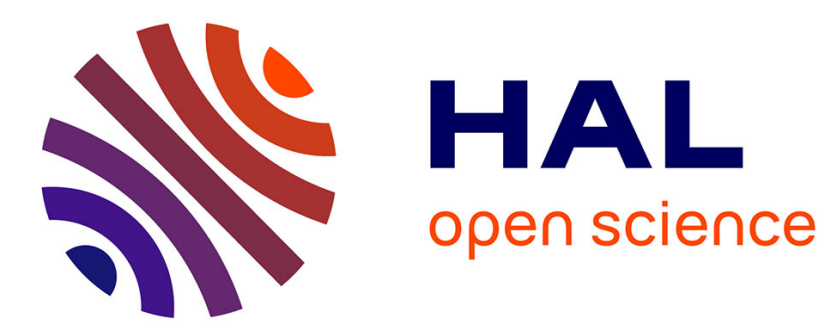

\title{
Game Theory for Dynamic Spectrum Access
}

Samir M. Perlaza, Merouane Debbah, Samson Lasaulce, Jean Marie Chaufray

\section{To cite this version:}

Samir M. Perlaza, Merouane Debbah, Samson Lasaulce, Jean Marie Chaufray. Game Theory for Dynamic Spectrum Access. Cognitive Radio Networks: Architectures, Protocols and Standards, CRC Press, pp.259-290, 2010. hal-00448798

\section{HAL Id: hal-00448798 \\ https://hal-centralesupelec.archives-ouvertes.fr/hal-00448798}

Submitted on 20 Jan 2010

HAL is a multi-disciplinary open access archive for the deposit and dissemination of scientific research documents, whether they are published or not. The documents may come from teaching and research institutions in France or abroad, or from public or private research centers.
L'archive ouverte pluridisciplinaire HAL, est destinée au dépôt et à la diffusion de documents scientifiques de niveau recherche, publiés ou non, émanant des établissements d'enseignement et de recherche français ou étrangers, des laboratoires publics ou privés. 


\section{Editors}

November 5, 2008 


\section{Contents}

1 Game Theory for Dynamic Spectrum Access 1

1.1 Introduction . . . . . . . . . . . . . . . . . . . . . . 2

1.2 Formulation of the DSA Problem . . . . . . . . . . . . . . 4

1.2.1 DSA in Multiple Access Channels . . . . . . . . . . . . . 5

1.2.2 DSA in Interference Channels . . . . . . . . . . . . . . . . 6

1.2.3 General Assumptions . . . . . . . . . . . . . . . . . 8

1.3 Dynamic Spectrum Access as a Game . . . . . . . . . . . . . . . . . . . . 8

1.3.1 The Game Model . . . . . . . . . . . . . . . . . . . . . . . 8

1.3.2 Non-Cooperative and Cooperative Games . . . . . . . . . . . . . . 9

1.3.3 The Nash Equilibrium Concept . . . . . . . . . . . . . . . . . . 10

1.3.4 Optimality Measures . . . . . . . . . . . . . . . 10

1.4 Open Spectrum Access Games . . . . . . . . . . . . . . . . . . 11

1.4.1 Formulation of the Game . . . . . . . . . . . . . . . . . 12

1.4 .2 Single Stage Games . . . . . . . . . . . . . . . . . . . . 14 
1.4.3 Repeated Games . . . . . . . . . . . . . . . . . . . . . . . 19

1.4.4 Bayesian Games . . . . . . . . . . . . . . . . . . . . . . . 23

1.4.5 Coalitional Games . . . . . . . . . . . . . . . . . 26

1.5 Hierarchical Spectrum Access Games . . . . . . . . . . . . . . . . . 27

1.5.1 Stackelberg Games . . . . . . . . . . . . . . . . . . . . . 28

1.6 Concluding Remarks . . . . . . . . . . . . . . . . . . . . . . 32 


\section{Chapter 1}

\section{Game Theory for Dynamic Spectrum}

\section{Access}

Samir Medina Perlaza, France Telecom R\&D - Orange Labs Paris, France.

Samson Lasaulce, Laboratoire des Signaux et Systèmes (LSS) - CNRS - SUPELEC, France.

Mérouane Debbah, Alcatel Lucent Chair in Flexible Radio, SUPELEC, France.

Jean-Marie Chaufray, France Telecom R\&D - Orange Labs Paris, France.

In this chapter, the competitive interaction of radio devices dynamically accessing the spectrum is studied using tools from game theory. Depending on the scenario under consideration, the dynamic spectrum access (DSA) is modeled by different types of games following both a non-cooperative and a cooperative approach. In the first case, each radio device aims to selfishly maximize an individual performance metric (e.g., individual data rate), while in the second case such maximization concerns global network parameters (e.g., network sum-rate). In each case, we analyze network equilibria, which allow network designers, operators, or manufactures to predict the behavior and performance of cognitive networks and/or terminals. 


\section{$1.1 \quad$ Introduction}

For many years, management of the radio spectrum has been based on the classic property right model. In this model, operators are granted licenses which allow them to exclusively use a certain frequency band in a given area for a specific service [3]. However, results from recent studies [1] show that this model often leads to an inefficient usage of the spectrum in terms of spectral efficiency (bits/Hz) due to two main reasons. First, the spectrum remains unused during the time that the licensed (primary) systems are idle, and second, the spectrum can be congested in one area while it remains unused in another due to a low spatial density of radio devices.

In this chapter, we consider the efficient use of the radio spectrum and analyze dynamic spectrum access (DSA) as a paradigm to improve the spectral efficiency of multiple communications systems subject to mutual interference. Here, we consider DSA as either a dynamic spectrum allocation in the case where a central controller exists or a spontaneous access where cognitive radios (CR) [2] autonomously decide to access the spectrum in a scenario where no central control is present. CRs are radio devices equipped with sensing systems that allow them to be aware of the environment they are operating in. More specifically, they are able to identify other active radios and estimate unused radio resources (frequency bands, time slots, spreading sequences, spatial directions, etc.). Additionally, such devices are able to self-adapt (in terms of coding-modulation scheme, power allocation, etc.) to compete with other devices and use the radio spectrum more efficiently.

There are at least two scenarios of high practical interest for the study of DSA [4]: Hierarchical spectrum access (HSA) and open spectrum access (OSA). In HSA, CRs coexist with legacy systems if and only if the additional interference overcome by the pre-existing systems is below a specific threshold. Such thresholds can be predefined by network operators, manufacturers or regulation entities to ensure certain quality of service (QoS) in the primary systems. In this scenario, CRs only transmit using radio resources left unused by the primary systems. Hence, CRs are often called opportunistic or secondary radio devices. Such unused radio resources are called spectrum access opportunities (SAO) or available channels. Typically, an available channel consists of non-occupied time slots in time division multiple 
access (TDMA), frequency bands in frequency division multiple access (FDMA), spatial directions in spatial division multiple access (SDMA), tones in orthogonal frequency division multiple access (OFDMA), or spreading codes in code division multiple access (CDMA). In HSA, once the SAOs have been identified each CR decides whether to transmit based on their own performance criterion.

In OSA, the notion of primary and secondary systems does not exist, at least in its conventional definition. In this scenario, each terminal has the same rights to access the spectrum at any time. OSA typically includes the case of unlicensed bands (e.g., the industrial, scientific and medical (ISM) band [2.400, 2.500] GHz). Radio devices operating in these bands include cordless telephones, wireless sensors, and devices operating under the standards of Wi-Fi (IEEE 802.11), Zig-Bee (IEEE 802.15.4), and Bluetooth (IEEE 802.15.1). Here, each technology implements different coding and modulation schemes, so there exists neither a common multiple access (MA) technique nor a signaling system to harmonize the use of these bands. Different governmental agencies, such as such as the European Radio Communications Office (ERO) in Europe and the Federal Communications Commission (FCC) in the USA, have defined a set of few rules either in terms of power spectral density masks or in terms of time duty cycles, depending on the application [6]. The power spectral density mask define the limits on the peak-to-average power ratio (PAPR) as a function of the frequency offset around the central frequency. The time duty cycle defines the longest cumulative period a specific device is allowed to transmit within a time unit.

In this chapter, we assume that CRs can sense their environment in a sufficiently reliable manner so that SAOs can be perfectly identified. Then, if SAOs are known by each CR, the common problem with either HSA or OSA is that there is a group of terminals competing for spectral resources. The rules the terminals must follow may differ, but in each case, it is a problem of interactions between cognitive entities that must make decisions to optimize their performance metrics. Hence, game theory, a branch of mathematics which studies the interaction between several decision-makers, is the dominant paradigm to analyze such problems [7]. Such analysis will be the focus of this chapter.

This chapter is organized as follows, in Sec. 1.2 we describe the problem of DSA and 
identify several scenarios depending on the network topology. More specifically, we analyze the DSA problem in both the multiple access channel (MAC) and interference channel (IC). In Sec. 1.3 we present fundamental concepts of game theory used to study DSA. Here, we model the DSA problem as games following both cooperative and non-cooperative approaches. In Sec. 1.4 we focus on open spectrum access (OSA) games, which includes the case of unlicensed bands. Here, we study how non-cooperative games often lead to suboptimal equilibrium points and present several techniques to improve the game outcomes. The two approaches presented for including a certain degree of cooperation between the players, and therefore improving the equilibrium efficiency are the repetition of the game (repeated games) and coalitions between several terminals to jointly compete against other coalitions (coalitional games). In Sec. 1.5 we discuss games modeling the co-existence between primary systems and opportunistic systems (hierarchical spectrum access (HSA)). Here, another technique to improve the game outcome on non-cooperative games is studied. We introduce a certain degree of hierarchy among the terminals (Stackelberg games) regarding for instance, either their nature (primary or secondary radio devices) or their decoding order at the receiver. Finally, in Sec. 1.6 we present open issues related to both OSA and HSA problems and state our conclusions.

\subsection{Formulation of the DSA Problem}

Dynamic access to the radio spectrum suggests either a dynamic allocation of SAOs (if a central controller exists) or a spontaneous access (if no central controller exists). However, two or more radio devices transmitting on the same channel (using the same SAO) might either degrade or break the communication off due to mutual interference. Hence, the formulation of the DSA problem can be summarized by the question: How does one optimally design the system to let each radio device (not necessarily a CR) use the available channels while maximizing the spectral efficiency i.e., the number of successfully transmitted bits per second over total available bandwidth?

In this chapter we constraint the study of DSA to the choice of the best channels (SAO) 
and the optimal transmit powers per channel. It should be noted that other degrees of freedom such as modulation-coding schemes, constellation size, polarization, type of receiver, etc. might also be considered to increase the spectral efficiency [58, 59].

Regarding the topology, we focus on both the multiple access channel (MAC) and the interference channel (IC). In the former case, several radio devices transmit to a single receiver. In the latter, each radio device transmits to a different receiver. This apparent simple difference between IC and MAC immediately implies different constraints regarding the channel state information (CSI), interference cancelation, and moreover, signaling among the active users in the network.

\subsubsection{DSA in Multiple Access Channels}

The multiple access channel, also known as the many-to-one channel, consists of $K$ transmitters aiming to communicate with a single receiver using a common channel [39]. If $N \geqslant 1$ channels are available, then there exists $N$ independent or parallel MACs, where transmitters in different MACs do not interfere each other. For instance, this model corresponds to the uplink channel in a single-cell multi-carrier cellular system.

Regarding notational aspects, the channel gain from transmitter $i$ to the receiver over the channel $n$ is denoted by $h_{i}^{n}$. We assume a block flat-fading channel model such that channel realizations remain constant during the transmission of $M$ consecutive symbols. All the channel realizations, $\forall i=\{1, \ldots, K\}$ and $\forall n=\{1, \ldots, N\}$ are drawn from a Gaussian distribution with zero mean and unit variance. The power allocated by transmitter $i$ to channel $n$ is denoted by $p_{i}^{n}$. Each transmitter is power-limited, i.e., for the $i^{\text {th }}$ transmitter, its transmit power cannot exceed $p_{i, \max }$, i.e., $\forall i \in\{1, \ldots, K\}, \quad \sum_{n=1}^{N} p_{i}^{n} \leqslant p_{i, \max }$.

The symbol sent by transmitter $i$ over channel $n$ is represented by $x_{i}^{n}$. We consider that transmitted symbols $\forall i=\{1, \ldots, K\}$ and $\forall n=\{1, \ldots, N\}$, are random variables with zero mean and unit variance. The noise at the receiver is denoted by $w$ and corresponds to an additive white Gaussian noise (AWGN) process with zero mean and variance $\sigma^{2}$. In vector 
notation, the channel realizations are written as $\boldsymbol{h}_{i}=\left(h_{i}^{1}, \ldots, h_{i}^{N}\right)$. Using a similar notation, the transmit powers, transmitted symbols, and noise are written as $\boldsymbol{p}_{i}=\left(p_{i}^{1}, \ldots, p_{i}^{N}\right), \boldsymbol{x}_{i}=$ $\left(x_{i}^{1}, \ldots, x_{i}^{N}\right)$, and $\boldsymbol{w}=\left(w^{1}, \ldots, w^{N}\right)$, respectively. Then, the received signal sampled at the symbol rate can be expressed as:

$$
\forall t \in\{1, \ldots, M\}, \quad \boldsymbol{r}_{i}(t)=\boldsymbol{h}_{i} \odot \sqrt{\boldsymbol{p}_{i}} \odot \boldsymbol{x}_{i}(t)+\sum_{j \neq i}^{K} \boldsymbol{h}_{j} \odot \sqrt{\boldsymbol{p}_{j}} \odot \boldsymbol{x}_{j}(t)+\boldsymbol{w}_{i}(t)
$$

The notation $\boldsymbol{a} \odot \boldsymbol{b}$ represents the Hadamard product of two vectors $\boldsymbol{a}$ and $\boldsymbol{b}$. According to this signal model, the received signal to interference plus noise ratio (SINR) on channel $n$ for transmitter $i$, denoted by $\gamma_{i}^{n}$ for all $i \in\{1, \ldots, K\}$ and for all $n \in\{1, \ldots, N\}$, is

$$
\gamma_{i}^{n}=\frac{p_{i}^{n}\left|h_{i}^{n}\right|^{2}}{\sigma^{2}+\sum_{j \neq i}^{K} p_{j}^{n}\left|h_{j}^{n}\right|^{2}} .
$$

In the MAC model the receiver knows the codebooks used by all the transmitters in the network [39]. This allows the receiver to use techniques such as multi-user detection and interference cancelation [38]. Additionally, the receiver is able to estimate the channel of all the transmitters. Conversely, without any additional signaling, each transmitter is able to estimate only its own channel. This fact implies that if the receiver is equipped with enough processing capabilities, the network performance could be optimized at the receiver and optimal transmission parameters (e.g., power allocation, modulation-coding scheme, etc.) can be fed back to the transmitters by using a control signaling protocol. The same procedure will be constrained at each transmitter due to the lack of knowledge about the other transmitter's channel gains. As described later, the availability of complete channel state information plays a key role in the DSA problem.

\subsubsection{DSA in Interference Channels}

The interference channel, known also as the many-to-many channel, consists of a set of $K$ point-to-point links close enough to produce mutual interference due to the co-existence on 
the same channel. If $N \geqslant 1$ channels are available, then there exists $N$ independent or parallel ICs, where transmitters in different ICs do not interfere each other. This topology typically appears in self-organized networks (adhoc networks) where nodes communicate in pairs, possibly using the same set of carriers.

To describe the IC model we keep the same notation and assumptions presented in the MAC case (Sec. 1.2.1). Only a slight modification is introduced to denote the channel realization from transmitter $i$ to receiver $j$ on channel $n$. Here, it is denoted by $h_{i j}^{n}$, with $n \in\{1, \ldots, N\}$ and $(i, j) \in\{1, \ldots, K\}^{2}$. The noise at receiver $i$ over channel $n$ is denoted by $w_{i}^{n}$ and $\boldsymbol{w}_{\boldsymbol{i}}=\left(w_{i}^{1}, \ldots, w_{i}^{N}\right)$. The received signal sampled at the symbol rate at the receiver $i$, denoted by $r_{i}^{n}(t)$ at sampling instant $t$, is written in a vector form as

$$
\forall t \in\{1, \ldots, M\}, \quad \boldsymbol{r}_{i}(t)=\boldsymbol{h}_{i i} \odot \sqrt{\boldsymbol{p}_{i}} \odot \boldsymbol{x}_{i}(t)+\sum_{j \neq i}^{K} \boldsymbol{h}_{j i} \odot \sqrt{\boldsymbol{p}_{j}} \odot \boldsymbol{x}_{j}(t)+\boldsymbol{w}_{i}(t),
$$

where the $N$-dimensional vector $\boldsymbol{h}_{\boldsymbol{i j}}$, for all $(i, j) \in\{1, \ldots, K\}^{2}$ is $\boldsymbol{h}_{i j}=\left\{h_{i j}^{1}, \ldots, h_{i j}^{N}\right\}$. The expression of the SINR on channel $n$ at the receiver $i$, denoted by $\gamma_{i}^{n}$ for all $i \in\{1, \ldots, K\}$ and for all $n \in\{1, \ldots, N\}$, is

$$
\gamma_{i}^{n}=\frac{p_{i}^{n}\left|h_{i, i}^{n}\right|^{2}}{\sigma^{2}+\sum_{j \neq i}^{K} p_{j}^{n}\left|h_{j, i}^{n}\right|^{2}}
$$

Often, in the IC model, each point-to-point link uses different codebooks which constraints the usage of multi-user detection techniques, and interference cancelation [38]. Moreover, each receiver is able to estimate only its own channel realization due to the usage of different training sequences in each link. Thus, the DSA problem turns out to be more challenging than in the MAC case due to the lack of knowledge about the channel gains of all the other transmitters. 


\subsubsection{General Assumptions}

For both types of network topologies, we assume that all the transmitters have perfect channel state information (CSI), i.e., each transmitter knows the channel realizations $\boldsymbol{h}_{i}$ for all $i \in\{1, \ldots, K\}$ in the MAC case, and $\boldsymbol{h}_{i, j}$ for all $(i, j) \in\{1, \ldots, K\}^{2}$ in the IC case. For ease of presentation but may be with loss of generality, we assume that all the radio devices are limited by the same power constraint $p_{\max }$, i.e., $\forall i \in \mathcal{J}, \quad p_{i, \max }=p_{\max }$. Additionally, we assume that all the transmitters are aware of such common maximum power threshold.

\subsection{Dynamic Spectrum Access as a Game}

In this section we present the fundamental concepts of game theory which will be used to study the problem of DSA. First, we introduce the concept of strategic form games and we present the cooperative and non-cooperative approaches. Afterwards, we introduce the concept of Nash equilibrium in non-cooperative games and optimality measures such as Pareto optimality and price of anarchy (PoA). Finally, a formulation of the DSA problem in game-theoretic terms is provided.

\subsubsection{The Game Model}

As mentioned above, game theory provides a natural mathematical framework to analyze strategic interactions between several decision makers. The set of rules governing such interaction is a game. The simplest representation of a game is the normal form. In normal form, a game is defined as follows:

Definition 1.3.1 (Normal Form Game) A game in normal form is denoted by $\left\{\mathcal{K}, \mathcal{S},\left\{u_{k}\right\}_{\forall k \in \mathcal{K}}\right\}$ and is composed of three elements

- a set of players: $\mathcal{K}=\{1, \ldots, K\}$;

- a set of strategy profiles: $\mathcal{S}=\mathcal{S}_{1} \times \ldots \times \mathcal{S}_{K}$, where $\mathcal{S}_{k}$ is the strategy set of the $k^{\text {th }}$ player; 
- a set of utility functions: The $k^{\text {th }}$ player's utility function is $u_{k}: \mathcal{S} \rightarrow \mathbb{R}_{+}$and is denoted by $u_{k}\left(s_{k}, \boldsymbol{s}_{-k}\right)$ where $s_{k} \in \mathcal{S}_{k}$ and $\boldsymbol{s}_{-k}=\left(s_{1}, \ldots, s_{k-1}, s_{k+1}, \ldots, s_{K}\right) \in \mathcal{S}_{1} \times \ldots \times \mathcal{S}_{k-1} \times$ $\mathcal{S}_{k+1} \times \ldots \mathcal{S}_{K}$.

The set of players is a finite set $\mathcal{K} \subset \mathbb{N}$ of which each element represents a player. The strategy set $S_{k}$ contains the set of actions player $k$ might take in the game. The utility function $u_{k}\left(s_{k}, \boldsymbol{s}_{-k}\right)$ allows a player to evaluate the convenience of its strategy $s_{k}$ with respect to the other players' strategies $\boldsymbol{s}_{-k}$.

Dynamic spectrum access can be modeled as a game (Sec. 1.4.1), when each transmitter is a player, the choice of its transmitting parameters is its strategy, and its utility function is described either in terms of its individual or network quality of service (QoS) parameters. The choice of a utility function leads to two different kind of games: non-cooperative and cooperative games.

\subsubsection{Non-Cooperative and Cooperative Games}

In a non-cooperative game, each player is selfish and unconcerned about all the other players' performance. Each terminal chooses its strategy to optimize its own performance metric under the assumption that all players are rational and adopt the same selfish behavior [24]. Thus, each player's utility function is defined in terms of local QoS targets (e.g., individual transmission rate). In a cooperative approach, each player aims at maximizing a common benefit for the set of players assuming that all the other players have adopted the same cooperative behavior. A common benefit could be interpreted for instance, as the sum of individual benefits (social welfare problem).

Often, if the performance metric (utility function) is well chosen, non-cooperative games might be played by each player using only local information (e.g., channel gains and power constraints regarding only a given player). However, cooperative games often require information regarding all the players' local information. Hence, cooperative games are often used either when there exists a central controller (e.g., a base station) which has complete information about all the players or when communications among all the players is possible 
(e.g., common signaling is available and affordable) $[32,33]$. In the next sections, we study some of the features of cooperative and non-cooperative games required for our discussions on DSA in Sec. 1.4 and Sec. 1.5.

\subsubsection{The Nash Equilibrium Concept}

An important concept in non-cooperative game theory is the Nash equilibrium (NE) [54, 53]. An NE corresponds to a profile of strategies $\boldsymbol{s}^{*}=\left(s_{1}^{*}, \ldots, s_{K}^{*},\right)$ for which each player's strategy $s_{k}^{*}, \forall k \in \mathcal{K}$ is the optimal response to all the other players' strategies $\boldsymbol{s}_{-k}^{*}$.

Definition 1.3.2 (Nash Equilibrium) In the game $\left\{\mathcal{K}, \mathcal{S},\left(u_{k}\right)_{\forall k \in \mathcal{K}}\right\}$, a strategy profile $\boldsymbol{s}^{*}=\left(s_{1}^{*}, \ldots, s_{K}^{*}\right) \in \mathcal{S}$ is an $N E$ if it satisfies,

$$
\forall k \in \mathcal{K} \text { and } \forall s_{k} \in \mathcal{S}_{k}, \quad u_{k}\left(s_{k}^{*}, \boldsymbol{s}_{-k}^{*}\right) \geqslant u_{k}\left(s_{k}, \boldsymbol{s}_{-k}^{*}\right)
$$

That is, at the NE, any unilateral deviation from the strategy profile $\boldsymbol{s}^{*}$ of player $k$, $\forall k \in \mathcal{K}$ will not increase its utility function $u_{k}$. Hence, at the NE there does not exist any motivation for a player to deviate from the NE strategy profile [54, 53, 17]. As players are selfish and decide by themselves their strategy, one question arises: does an NE lead to an efficient game outcome?

\subsubsection{Optimality Measures}

The NE outcome is a stable solution to unilaterally deviations, however it might not be optimal $[17,31]$. A formal measure of any game outcome's optimality is the Pareto optimality. A Pareto optimal strategy profile can be described as follows:

Definition 1.3.3 (Pareto Optimality) In the game $\left\{\mathcal{K}, \mathcal{S},\left\{u_{k}\right\}_{\forall k \in \mathcal{K}}\right\}$, let $s=\left(s_{1}, \ldots, s_{K}\right)$ 
and $\boldsymbol{s}_{i}^{\prime}=\left(s_{1}^{\prime}, \ldots, s_{K}^{\prime}\right)$ be two different strategy profiles in $\mathcal{S}$. Then, if

$$
\forall k \in \mathcal{K} \quad u_{k}\left(s_{k}, s_{-k}\right) \geqslant u_{k}\left(s_{k}^{\prime}, \boldsymbol{s}_{-k}^{\prime}\right)
$$

with strict inequality for at least one player, the strategy profile $\boldsymbol{s}$ is Pareto-superior to the strategy profile $\boldsymbol{s}_{i}^{\prime}$. If there exists no strategy that is Pareto superior to $\boldsymbol{s}_{i}$, then $\boldsymbol{s}_{i}$ is Pareto optimal.

Often, the NE strategy profile is not Pareto optimal and the loss of performance observed in a non-cooperative game due to the lack of cooperation is a common optimality measure called the price of anarchy (PoA) [37].

Definition 1.3.4 (Price of Anarchy) Let the triplet $\left\{\mathcal{K}, \mathcal{S},\left\{u_{k}\right\}_{\forall k \in \mathcal{K}}\right\}$ be a non-cooperative game and let $\boldsymbol{s}^{*}=\left(s_{1}^{*}, \ldots, s_{K}^{*}\right)$ be the $N E$ strategy profile which minimizes (if several $N E s$ ) $\sum_{k=1}^{K} u_{k}\left(s_{k}^{*}, s_{-k}^{*}\right)$. Then, the ratio

$$
\mathrm{PoA}=\frac{\max _{\boldsymbol{s} \in \mathcal{S}} \sum_{k=1}^{K} u_{k}\left(s_{k}, \boldsymbol{s}_{-k}\right)}{\sum_{k=1}^{K} u_{k}\left(s_{k}^{*}, \boldsymbol{s}_{-k}^{*}\right)}
$$

is the price of anarchy (PoA) of the game $\left\{\mathcal{K}, \mathcal{S},\left\{u_{k}\right\}_{\forall k \in \mathcal{K}}\right\}$.

\subsection{Open Spectrum Access Games}

In this section we study the DSA problem following the OSA model described in Sec. 1.1. As stated before, the OSA scenario is typical of non licensed bands where there exists neither common signaling due to the use of different communications standards (Wi-Fi, Zigbee, etc), nor uniform QoS requirements, due to the different applications (voice, video, data, etc.) radio devices are used for. We present a simple game to model such interactions. 
Afterwards, we present existing results using this model, namely existence of NE points and its optimality analysis.

\subsubsection{Formulation of the Game}

We model the interaction between $K$ radio devices in the OSA model sharing $N$ orthogonal channels as a strategic game. We study the different variants of such a game considering the topology of the network (MAC or IC), as well as the goals of each player (cooperative or non-cooperative). This interaction can be defined as follows,

Definition 1.4.1 (OSA Game in Normal Form) In normal form, the OSA game is denoted by $\left\{\mathcal{K}, \mathcal{S},\left\{u_{k}\right\}_{\forall k \in \mathcal{K}}\right\}$, where each of the elements of $\mathcal{K}=\{1, \ldots, K\}$, represents one of the $K$ transmitters. The strategy set is $\mathcal{S}=\mathcal{S}_{1} \times \ldots \times \mathcal{S}_{N}$, where $\mathcal{S}_{i}$ is the strategy set of player $i$ :

$$
\mathcal{S}_{i}=\left\{\boldsymbol{p}_{i}=\left(p_{i}^{1}, \ldots, p_{i}^{N}\right): \forall n \in\{1, \ldots, N\}, p_{i}^{n}>0 \text { and } \sum_{n=1}^{N} p_{i}^{n} \leqslant p_{\max }\right\}
$$

The utility function for the $i^{\text {th }}$ player is $u_{i}\left(\boldsymbol{p}_{i}, \boldsymbol{p}_{-i}\right)$ and it is defined differently depending on the topology of the network (e.g., MAC or IC) and the approach (cooperative or noncooperative) adopted by the players.

Later, we refine this definition by specifying the utility function for each particular case of study (MAC/IC and cooperative/non-cooperative).

\section{The Choice of the Utility Function}

In the current literature, the utility functions are often defined as a function of either the achieved data rate or as the ratio between the successfully transmitted bits per second (goodput) and the total transmit power. In our discussion we call the former case rateefficient $O S A$ and the latter energy-efficient $O S A$. 
The utility functions in the rate-efficient OSA game following a non-cooperative approach can be defined as

$$
\forall k \in \mathcal{K}, \quad u_{k}(\boldsymbol{p}, \boldsymbol{h})=\sum_{n=1}^{N} \log \left(1+\gamma_{k}^{n}\right) .
$$

which corresponds to the sum data rate achieved by player $k$ over all its available channels. Here, $\boldsymbol{p}$ is a $N K$-dimensional vector obtained by concatenating the vectors $\boldsymbol{p}_{i} \forall i \in \mathcal{K}$. The vector $\boldsymbol{h}$ is defined differently in the MAC and IC games. In the MAC game it is a $N K$ dimensional vector obtained by concatenating $K N$-dimensional vectors $\boldsymbol{h}_{i} \forall i \in \mathcal{K}$. In the IC game, it corresponds to a $N K^{2}$-dimensional vector obtained by concatenating the $K^{2}$ $N$-dimensional vectors $\boldsymbol{h}_{i, j} \forall(i, j) \in \mathcal{K}^{2}$. The $\operatorname{SINR} \gamma_{i}^{n}$ is either the expression (1.2) for the MAC case or expression (1.4) for the IC case.

The utility function in the energy-efficient OSA games is defined as

$$
u_{k}(\boldsymbol{p}, \boldsymbol{h})=\frac{\sum_{n=1}^{N} R_{k}^{n} f\left(\gamma_{k}^{n}\right)}{\sum_{n=1}^{N} p_{k}^{n}}
$$

The data rate $R_{k}^{n}$ is fixed by the particular modulation-coding scheme used by transmitter $k$ on channel $n$. In the sequel, we assume that all transmitters are subject to the same rate $R$ over all the channels. The function $f\left(\gamma_{i}^{n}\right): \mathbb{R}_{+} \rightarrow[0,1]$, known in the literature as the efficiency function, is assumed to be sigmoidal function that approximates the fraction of successfully transmitted bits per frame given the SINR $\gamma_{i}^{n}$ over the channel $n$. A typical approximation of the $f($.$) function is$

$$
f(\gamma)=\left(1-e^{\gamma}\right)^{M}
$$

where $\gamma$ represents a given SINR and $M$ is the framelength. The SINR $\gamma_{i}^{n}$ in (Eq. 1.10) might be either expression (1.2) for the MAC case or expression (1.4) for the IC case. Since the rates $R_{k}^{n}$ are constants and might be the same for all the transmitters, we ignore them 
and consider the utility function (Eq. 1.10) as the ratio $\frac{\sum_{n=1}^{N} f\left(\gamma_{k}^{n}\right)}{\sum_{n=1}^{N} p_{k}^{n}}$ in the sequel.

The utility function defined in (Eq. 1.10) is measured in bits/s per Joule. It describes how many bits can be successfully transmitted per Joule drained from the battery. This is why the model is known as an energy-efficient model. Extensive discussions on this utility function are presented in [15].

In the cooperative game, more specifically in either the MAC or IC cooperative game, and in either the rate-efficient or energy-efficient case, the cooperative utility function is defined as

$$
U(\boldsymbol{p}, \boldsymbol{h})=\sum_{k=1}^{K} u_{k}(\boldsymbol{p}, \boldsymbol{h}),
$$

where $u_{k}(\boldsymbol{p}, \boldsymbol{h})$ is the utility function of the player $k$ in the non-cooperative game.

In the following sections we study the current results in OSA games using the general OSA game described in Def. 1.4.1 with the utility functions (Eq. 1.10) and (Eq. 1.9). Other utility functions are also studied in later sections.

\subsubsection{Single Stage Games}

In the current literature the OSA problem has been analyzed considering the game defined in 1.4.1 for both MAC and IC channel. For the energy-efficient utility function, Meshkati $[15,48]$ has extensively studied the MAC channel. The IC case has been investigated by Etkin $[35,36]$ in the case of unlicensed bands inspired by previous results from Yu $[50,51]$ and Chung [52] that discuss such games considering the rate-efficient utility function. 


\section{MAC Single Stage Games}

Consider the definition of the OSA game (Def. 1.4.1) with a utility function defined by (Eq. 1.10) for the MAC case. A special case of this utility is the single carrier CDMA scenario, where $N=1$. For such particular case, the utility function reduces to

$$
\forall k \in \mathcal{K}, \quad u_{i}\left(p_{i}, \boldsymbol{p}_{-i}\right)=\frac{R_{i} f\left(\gamma_{i}\right)}{p_{i}}
$$

This utility function exhibits several properties which are carefully studied by Rodriguez in [49]. Based on such study, it is known that an NE is observed if all the transmitters achieve a SINR as close as possible to an optimal value denoted $\gamma^{*}$. Such optimal SINR is the unique solution to the equation

$$
\gamma^{*} f^{\prime}\left(\gamma^{*}\right)-f\left(\gamma^{*}\right)=0
$$

which corresponds to the unique maximum of the function $f($.$) (Eq. 1.13).$

If all the players attempt to achieve the same $\operatorname{SINR} \gamma^{*}$. The optimal power value is:

$$
p_{i}^{*}=\frac{\sigma^{2}}{\left|h_{i}\right|^{2}} \frac{\gamma^{*}}{1-\alpha(K-1) \gamma^{*}},
$$

where $\alpha$ is the inverse of the spreading length $S, \alpha=\frac{1}{S}$ in CDMA. For all $i$, if the transmit power $p_{i}^{*}$ required to achieve $\gamma^{*}$ is higher than $p_{\max }$, then transmitting at $p_{i}^{*}=p_{\max }$ is also a Nash Equilibrium. Thus, for the CDMA case $(N=1)$ the existence and uniqueness of the NE is always ensured [48].

When other MA technique or multi-carrier CDMA is used $(N>1)$, even though the NE always exists, it is not always unique [15, 48]. First, we use the result obtained by Meshkati et al. [15] which states that

$$
\forall i \in \mathcal{K}, \quad \frac{f\left(\gamma^{*}\right)}{\sum_{n=1}^{N} p_{i}^{n}} \leqslant \frac{f\left(\gamma^{*}\right)}{p_{i}^{L *}}
$$


where, $L=\arg \min _{n=1, \ldots N}\left\{p_{i}^{n *}\right\}$ and $p_{i}^{n *}$ is obtained with

$$
p_{i}^{n *}=\frac{1}{\left|h_{i}^{n}\right|^{2}}\left(\frac{\gamma^{*} \sigma^{2}}{1-\alpha \Theta(n) \gamma^{*}}\right)
$$

where, $\Theta(n)$ represents the number of players simultaneously transmitting over channel $n$. Again, in the case where $p_{i}^{L *}>p_{\max }$, an NE is achieved by transmitting at $p_{i}^{L *}=p_{\max }$. The important remark here is that, at the NE each player uses only one of its available channels [15]. Nonetheless, for certain conditions over the channels gains, the NE is not always unique. To shed a light on the existence and uniqueness of the NE we consider the following example.

Example 1.4.1 (Two-Player-Two-Channel MAC Game) Consider the particular case of a 2-carrier $M A C(N=2)$ with only two active transmitters $(K=2)$. According to the discussion above, the optimal OSA strategy for each player is to transmit only over the subcarrier which requires the lowest transmit power level. In this respect, we can model this interaction as two players aiming at transmitting at the minimum power level required to achieve a SINR $\gamma^{*}$ on any of the channels. Here, their strategy is transformed into the choice to transmit over channel 1 (C1) or 2 (C2). Considering this new definition of the game, four particular scenarios might arise. Scenario (1) and (4): both users transmit over the first and second channel, respectively. Scenario (2): the player 1 transmits over the first channel and the player 2 transmits over the second channel. For the scenario (3) the converse applies. For the ease of presentation, we consider single user decoding (SUD) at the receiver.

Under the assumption that each player only decodes its own signal and treats the other player signal as noise, we calculate the required transmit power to achieve the optimal SINR $\gamma^{*}$ depending to the channel each player decides to transmit on. In Table 1.1 we present the possible transmit power levels in this game.

Based on the power levels shown in Tab. 1.1, we identify the best responses each of the players might take with respect to the other player's responses. Thus, an NE might be observed on any of the four scenarios depending on the channel gains, e.g., 


\begin{tabular}{|c|cc|cc|}
\hline & \multicolumn{2}{|c|}{ C1 } & \multicolumn{2}{c|}{ C2 } \\
\hline \multirow{2}{*}{ C1 } & $p_{1,1}=\frac{\sigma^{2} \gamma^{*}}{\left|h_{11}\right|^{2}\left(1-\gamma^{*}\right)}$ & $p_{1,2}=0$ & $p_{1,1}=0$ & $p_{1,2}=\frac{\sigma^{2} \gamma^{*}}{\left|h_{12}\right|^{2}}$ \\
& $p_{2,1}=\frac{\sigma^{2} \gamma^{*}}{\left|h_{21}\right|^{2}\left(1-\gamma^{*}\right)}$ & $p_{2,2}=0$ & $p_{2,1}=\frac{\sigma^{2} \gamma^{*}}{\left|h_{21}\right|^{2}}$ & $p_{2,2}=0$ \\
\hline \multirow{2}{*}{ C2 } & $p_{1,1}=\frac{\sigma^{2} \gamma^{*}}{\left|h_{11}\right|^{2}}$ & $p_{1,2}=0$ & $p_{1,1}=0$ & $p_{1,2}=\frac{\sigma^{2} \gamma^{*}}{\left|h_{12}\right|^{2}\left(1-\gamma^{*}\right)}$ \\
& $p_{2,1}=0$ & $p_{2,2}=\frac{\sigma^{2} \gamma^{*}}{\left|h_{22}\right|^{2}}$ & $p_{2,1}=0$ & $p_{2,2}=\frac{\sigma^{2} \gamma^{*}}{\left|h_{22}\right|^{2}\left(1-\gamma^{*}\right)}$ \\
\hline
\end{tabular}

Table 1.1: Transmit Power levels at each possible equilibrium point for the two-player-twocarrier game with single user decoding (SUD).

- Equilibrium 1: $\left(C_{1}, C_{1}\right)$

$$
\frac{\left|h_{12}\right|^{2}}{\left|h_{11}\right|^{2}}<1-\gamma^{*} \quad \text { and } \quad \frac{\left|h_{22}\right|^{2}}{\left|h_{21}\right|^{2}}<1-\gamma^{*}
$$

- Equilibrium 2: $\left(C_{1}, C_{2}\right)$

$$
\frac{\left|h_{12}\right|^{2}}{\left|h_{11}\right|^{2}}<\frac{1}{1-\gamma^{*}} \quad \text { and } \quad \frac{\left|h_{22}\right|^{2}}{\left|h_{21}\right|^{2}}>1-\gamma^{*}
$$

- Equilibrium 3: $\left(C_{2}, C_{1}\right)$

$$
\frac{\left|h_{12}\right|^{2}}{\left|h_{11}\right|^{2}}>1-\gamma^{*} \quad \text { and } \quad \frac{\left|h_{22}\right|^{2}}{\left|h_{21}\right|^{2}}<\frac{1}{1-\gamma^{*}}
$$

- Equilibrium 4: $\left(C_{2}, C_{2}\right)$

$$
\frac{\left|h_{12}\right|^{2}}{\left|h_{11}\right|^{2}}>\frac{1}{1-\gamma^{*}} \quad \text { and } \quad \frac{\left|h_{22}\right|^{2}}{\left|h_{21}\right|^{2}}>\frac{1}{1-\gamma^{*}} .
$$

In Fig. 1.1 we plot such conditions and the regions where the different NE are observed. Therein, it is evident that the existence of the NE is always ensured. Nonetheless, it might not be unique. Indeed, in the region where channel gains simultaneously satisfy $1-\gamma^{*} \leqslant$ $\frac{\left|h_{12}\right|^{2}}{\left|h_{11}\right|^{2}} \leqslant \frac{1}{1-\gamma^{*}}$ and $1-\gamma^{*} \leqslant \frac{\left|h_{22}\right|^{2}}{\left|h_{21}\right|^{2}} \leqslant \frac{1}{1-\gamma^{*}}$ the Nash equilibrium is not unique. In such region, both scenarios 3 and 2 are Nash equilibria.

Regarding this example (Eg. 1.4.2), an algorithm for achieving an NE was presented in [15]. However, such algorithm was unable to always converge to at least one NE, even though the existence of at least one NE is always ensured. A unique NE is observed if the receiver implements interference cancelation assuming that the same decoding order is adopted on all 


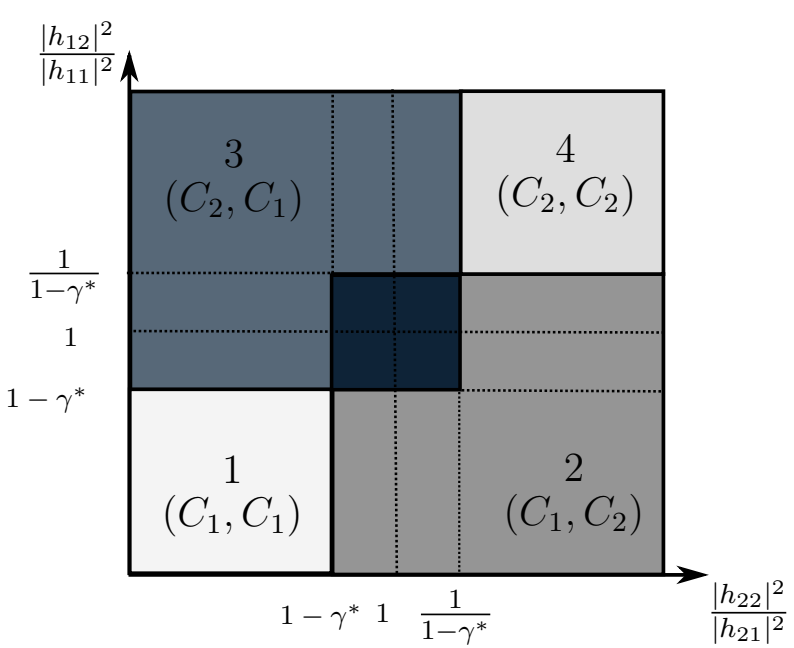

Figure 1.1: Regions of the possible Nash equilibria for the two-players two-carriers game with single user detection (SUD).

the available channels. Nonetheless, this new game requires the knowledge of the decoding order of all the players, which is necessarily a demanding task due to the required additional signalling.

\section{IC Single Stage Games}

In a cooperative context, if the utility function is the sum data rate of all the players as suggested in [35], Pareto optimal strategies coincide with the border of the capacity region. Unfortunately, the capacity region for the IC is still undetermined and is a subject of intensive research [39]. For simple cases, such as the high interference regime, i.e.,

$$
\forall n \in\{1, \ldots, N\} \text { and } \forall(i, j) \in \mathcal{K}^{2}, \quad\left|h_{i, j}^{n}\right|^{2}\left|h_{j, i}^{n}\right|^{2}>\left|h_{i, i}^{n}\right|^{2}\left|h_{j, j}^{n}\right|^{2}
$$

it is known that Pareto optimal sum data rates are achieved by using orthogonal channels, i.e., $\left\langle\boldsymbol{p}_{i}, \boldsymbol{p}_{j}>=0, \forall(i, j) \in \mathcal{K}^{2}\right.$. For instance, such optimal power profiles are obtained with frequency division multiplexing (FDM). This result is due to the fact that the condition in expression (1.22) leads to a concave optimization problem whose solution is easy to identify. If the high interference regime is not met (Eq. 1.22) then the Pareto optimal strategies require more complex calculations [35]. 
In the non-cooperative context, the utility functions correspond to the individual data rate achieved by each player over all its channels (Eq. 1.9). In this case, due to the selfish behavior of each player, we are interested on NE points. In [35], it has been shown that uniform power spreading among all the channels corresponds to an NE point. We denote such optimal strategy profile as $\boldsymbol{p}_{k}^{*}$, for the player $k$. Thus,

$$
\forall k \in \mathcal{K}, \quad \boldsymbol{p}_{k}^{*}=\frac{p_{\max }}{N} \mathbb{1}_{N}
$$

where $\mathbb{1}_{N}$ is a $N$-dimensional vector whose entries are all ones. This result comes from the fact that an optimal response for a given player given the strategies of all the other players is to waterfill [41] over the noise plus interference observed on its channels. This interaction leads to stable point which corresponds to spreading the total transmit power uniformly. However, the uniqueness of such NE point is not ensured. In [35] a sufficient condition for the uniqueness of such equilibrium is provided,

$$
\forall n \in\{1, \ldots, N\}, \quad \sum_{j=1}^{K} \frac{\left|h_{j i}^{n}\right|^{2}}{\left|h_{i i}^{n}\right|^{2}} \leqslant 1 .
$$

This result comes from the Karush-Kuhn-Tucker conditions of the optimization problem of the set of data rates. Less restrictive sufficient conditions for the uniqueness of the NE are provided in [41].

\subsubsection{Repeated Games}

When the interaction between the decision makers is modeled as a strategic game, as discussed in Sec. 1.3.1, it is assumed that players make their decisions simultaneously and once during the whole game. In DSA, this approach is suited to one of the following situations: 1. networks with low mobility and fixed topology; 2. study of a network over a short period of time; 3. the users' decisions over time are taken independently e.g., if the users's utility is chosen to be its instantaneous data rate or energy efficiency (over a block). However, radio devices interact for long periods in a constantly changing environment. To take into account such practical features, we can consider a sequence of strategic games related each other in 
time. This model is known in game theory as repeated games.

A repeated game is a special case of dynamic game [55]. It can be seen as a strategic game which is played consecutively a finite or infinite number of times. These games are respectively referred to as infinitely repeated and finite horizon repeated games [17]. At each time or stage, the same game is played. In this Section, we focus on infinitely repeated games. For such case, the utility for each player is chosen to be a weighted sum of its own instantaneous utility (e.g., Eq. (1.10) or (Eq. 1.9)) at stage $m, u_{k}(m)$ i.e.,

$$
r_{k}=(1-\delta) \sum_{m=0}^{\infty} \delta^{m} u_{k}(m)
$$

where $\delta$ is a normalization factor such that $0<\delta \leqslant 1$, and $r_{k}=1$ if $u_{k}=1$. The factor $\delta$ defines if the player is more interested either on the utility obtained at the initial stages of the game or the utility obtained in later stages.

At the end of each stage all the players observe the outcome of the game. For a transmitter to be able to directly observe the actions of the other transmitters, additional assumptions have to be made. For example, in a two-player game, if player 1 wants to evaluate the transmit power of player 2 from the signal received from the first player, some source separation algorithm and sufficiently accurate path loss model need to be assumed. In what follows, we assume that players can observe each other player's actions.

The OSA non-cooperative game described in Sec. 1.4.1 is modeled by an infinitely repeated game considering the utility function $r_{k}$ described in Eq. 1.25 where each $u_{k}(m)$, $\forall k \in \mathcal{K}$ and $\forall m \in \mathbb{N}$ corresponds to the individual data rate as defined in Eq. 1.9. In such a game, at each stage the same analysis presented in Sec. 1.4.2 applies. To extend such analysis to a repeated game, we resort the following definition.

Definition 1.4.2 (Perfect Sub-Game Nash Equilibrium) A set of strategies

$$
\boldsymbol{s}^{*}=\left(\boldsymbol{s}(t+1)^{*}, \ldots, \boldsymbol{s}(t+T)^{*}\right)
$$

with $\boldsymbol{s}(t)=\left(s_{1}(t), \ldots, s_{K}(t)\right)$ is a perfect sub-game (game from stage $t$ to state $t+T$ ) Nash 
Equilibrium if at every stage $t$ of the sub-game, the strategy $\boldsymbol{s}^{*}(t)$ is a Nash equilibrium.

In the single stage game discussed in Sec. 1.4.2 for the IC case with utility function (Eq. 1.9) an NE was obtained by uniformly spreading the transmit power among all the available channels. Then, in the repeated game, uniformly spreading the total power at each stage forms a perfect sub-game $N E \forall m \in \mathbb{N}$ [35]. Exploiting the fact that the game is infinitely repeated, an improvement based on the assumption that if a Pareto optimal strategy $\boldsymbol{p}^{\prime}$ (e.g., transmitting over orthogonal channels in the high interference regime) is achieved due to an initial coordination of the network, by means of a punishment policy, each transmitter is encouraged to keep the strategy $\boldsymbol{p}_{k}^{\prime}$ at each iteration $m$. If a player deviates from the strategy $\boldsymbol{p}^{\prime}$, the other players punish him by spreading the power over all the channels. A punishment will necessarily decrease the utility function of all the active players in the next stage [35]. Thus, no player is motivated to deviate from the initial agreement.

The strategy of punishments significantly improves the performance obtained with singlestage repeated games. In the repeated game, radio devices obtain Pareto optimal outcomes at each stage of the game. Playing a different strategy leads to a punishment which represents a lower utility function. This punishment policy can be seen as certain coordination between the players to improve the global performance (cooperative approach). It should be noted that no additional signaling is required once the players have set up their agreement.

Other application of repeated games to OSA is presented by Wendorf et al. in [25, 27]. Therein, the problem of dynamic frequency selection (DFS), typical from IEEE 802.11 networks is studied. The DFS problem can be defined as in Def. 1.4.1, where the set of strategies of each player is redefined as

$$
\forall i \in \mathcal{K}, \quad \mathcal{S}_{i}=\left\{p_{\max } \boldsymbol{e}_{1}, \ldots, p_{\max } \boldsymbol{e}_{N}\right\}
$$

where the $N$-dimensional vector $\boldsymbol{e}_{i}, \forall i \in \mathcal{K}$ is such that all its entries are zeros except the $i^{\text {th }}$ entry which is a one. In DFS each radio transmits only over a single channel and always at the maximum power. The analysis in $[25,27]$ considers only two players under the assumption that co-existence of both radio devices on the same channel leads to an outage condition due 
to mutual interference. This problem is modeled as a repeated game considering that at the starting point both players are using the same channel. Hence, a simplification is proposed and the strategy set for each player is reduced to the actions of either changing its channel (C) or staying (S) on the same channel. Thus $\forall k \in \mathcal{K}, \quad \mathcal{S}_{k}=\{S, C\}$. The utility function, which is in reality a cost function (players aim to minimize it), is defined as the sum of the delay (denoted by $v$ ) induced by switching from the current channel to a new one and the delay (denoted by $u$ ) due to the multiple access interference (which increases the probability of retransmission). In Tab. 1.4.3 the game under investigation is represented in its matrix form $[17,31]$. The first (resp. second) entry of the pair $(x, y)$ corresponds to the cost for user 1 (resp. 2).

\begin{tabular}{|r|c|c|}
\hline $1 / 2$ & $C$ & $S$ \\
\hline$C$ & $(v, v)$ & $(v, 0)$ \\
\hline$S$ & $(0, v)$ & $(1+u, 1+u)$ \\
\hline
\end{tabular}

Table 1.2: Game matrix for the DFS game [25]

The authors of $[25,27]$ do not link the strategies $S$ and $C$ to the channel being used and the channel to be used. Therefore, players randomly change to another channel. When the number of channels is large, the probability of overlapping is relatively small. Thus, the case of two players with two channels is the worse scenario in terms of probability of overlapping. Assuming a game with two channels and a certain probability (say $\rho$ ) that a user changes its channel, the authors of $[25,27]$ derived the solution of the game in terms of such probability. They provide the optimal probability $\rho^{*}$ a user should adopt during the stages of the game. The authors show that $\rho^{*}$ corresponds to a unique Nash equilibrium. The result is compared with a cooperative approach where the utility function is defined as the sum of the channel acquisition cost (delay) for each player. The comparison shows a significant loss in performance in terms of delay due to the lack of cooperation. This work has been extended to the case of several players in [26].

This work is important in the sense that it does not consider a set of actions as the strategy of each player. It rather defines the actions and assumes the strategy set as the probability with which each user will play each action in the repeated game. In game theory, this strategy definition is known as mixed-strategies [17]. 


\subsubsection{Bayesian Games}

So far, we have considered that each player has complete information about all the other players, i.e., each player knows all the other players' utilities. Regarding the DSA problem, this mean that each radio has perfect CSI and perfectly knows the power limitations of all the other radio devices. However, this assumption is quite far from the situation encountered in practice. In the open spectrum access model for example, it is more realistic to assume that a given radio transmitter only knows its own channel gains, QoS requirements and power limitations. In this Section, unless otherwise stated, each radio device only knows its own channel gain, power limitations and QoS requirements. Game theory provides a complete framework to analyze the interaction between decision makers with incomplete information. Bayesian Games are either static or dynamic games where at least one player does not know the utility function of one or more of its opponents. Thus, each user plays the game based on a probabilistic analysis. Therefore, these games are known as Bayesian games due to the Bayesian inference required to play.

Following the approach proposed by Harsanyi [42], a Bayesian game could be obtained by introducing some randomness in a strategic game. Suppose that there exists a set $\mathcal{K}=$ $\{1, \ldots, K\}$ of players with a set of actions $\mathcal{S}_{k}, \forall k \in \mathcal{K}$ and that each player does not know the utility function of all the other players. However, each player knows that there exists a finite set of possible types $\mathcal{T}_{i}$ for each player $k \in \mathcal{K}$. The corresponding type for each player is a random variable which follows a probability distribution known by all the players. Each type of player has a specific utility function and strategy set, denoted $u_{t}$ and $\mathcal{S}_{t}, \forall t i n \mathcal{T}$, respectively. Thus, the incomplete information game is transformed in a game where at least the probability distributions of the unknown parameters (e.g., utility functions and strategy sets) are known.

Definition 1.4.3 (Bayesian Game) A Bayesian game is completely described by the following set of parameters

- A set of $K$ players, $\mathcal{K}=\{1, \ldots, K\}$.

- A finite set of $T$ types of players $\mathcal{T}=\left\{t_{1}, \ldots, t_{T}\right\}$. 
- A probability density function of the different types of players: $\{f(t) \in[0,1] \mid \forall t \in \mathcal{T}\}$.

- A set of $T$ finite sets of strategies: $\mathcal{S}_{1}, \mathcal{S}_{2}, \ldots, \mathcal{S}_{T}$ each one for each type of player.

- A set of $T$ utility function $u_{k}: \mathcal{T} \times \mathcal{S} \rightarrow \mathbb{R}_{+}$, for each type of player.

The DSA problem presented in Sec. 1.2 can be modeled as a Bayesian game. Here, it could be stated that radio devices do not know the exact channel realizations of all the other players in the network and therefore, their utility function cannot be determined. Under a Bayesian formulation of the problem we can assume that players do not know the exact channel realization but it is known that it belongs to a known probability distribution. Under these assumptions we can now formulate the OSA problem following the definition Def. 1.4.1 considering that the set of types $\mathcal{T}$ corresponds to all the possible probability distributions which can model the channel realization of each player. For simplicity, we assume that all the channel gains follow the same distribution $f_{h}$.

A more realistic model would be to consider different probability distributions for each player. This is the case for example if some transmitters are in line of sight with their corresponding receivers (Rice channel distribution) while the other transmitters are linked with their receivers by Raleigh distributed channels.

The non-cooperative DSA problem in the IC case is studied in [43]. The underlying assumption is that radio devices do not know any of the parameters to play the game. More specifically, CSI is not available neither at the transmitter nor the receivers. Nonetheless, it is assumed that all the channel realizations are drawn from a known probability distribution $f_{h}$. The utility function for each player is chosen to be the expected value of the utility function defined in (1.9),

$$
\forall k \in \mathcal{K}, \quad \hat{u}_{k}\left(\boldsymbol{p}_{k}, \boldsymbol{p}_{-k}\right)=\mathbb{E}\left[u_{k}\left(\boldsymbol{p}_{k}, \boldsymbol{p}_{-k}\right)\right]
$$

where, the expectation is taken over the distribution $f_{h}$.

The authors in [43] analyze the problem from a non-cooperative point of view i.e., the goal of each user is to selfishly maximize its own utility function. Here, a highly desired 
outcome corresponds to a Nash equilibrium. When it exists, the optimal strategy profile $\boldsymbol{p}_{k}^{*}=\left\{p_{k}^{1 *}, \ldots, p_{k}^{N *}\right\}$ is a solution to the following optimization problem

$$
\forall k \in \mathcal{K}, \quad \boldsymbol{p}_{k}^{*}=\max _{\boldsymbol{p}_{k}} \int_{0}^{\infty} \hat{u}_{k}\left(\boldsymbol{p}_{k}, \boldsymbol{p}_{-k}\right) f_{h}(\boldsymbol{h}) \mathrm{d} \boldsymbol{h},
$$

The NE for this case is the transmit power vectors $\boldsymbol{p}^{*}=\left\{\boldsymbol{p}_{1}^{*}, \ldots, \boldsymbol{p}_{N}^{*}\right\}$, where

$$
\forall k \in \mathcal{K}, \quad \boldsymbol{p}_{k}^{*}=\frac{p_{\max }}{N} \mathbb{1}_{N}
$$

Thus, under the assumption of incomplete information, there is a unique NE at which all the transmitters uniformly spreading all the transmittable power between all the available channels. In a further step, the authors in [43] keep limited the number of players to $K=2$ and additionally restrict set of actions for each player such that in this new formulation, each player might assign either all the transmittable power to a unique channel or uniformly spread it over all the $N$ available channels. Therefore, the set $\mathcal{S}_{k}$ is made of $N+1$ vectors such that

$$
\forall k \in \mathcal{K}, \quad \mathcal{S}_{k}=\left\{p_{\max } \boldsymbol{e}_{1}, \ldots, p_{\max } \boldsymbol{e}_{N}, p_{\max } \mathbb{1}_{N}\right\}
$$

Moreover, it is assumed that the $i^{\text {th }}$ player perfectly knows the set of channels $\boldsymbol{h}_{i}=$ $\left(h_{i, i}, h_{-i, i}\right)$, i.e., its own channel and its interfering channels. As discussed before, the NE solution corresponds to the solution of the optimization problem,

$$
\forall k \in \mathcal{K}, \quad \boldsymbol{p}_{k}=\arg \max _{\boldsymbol{p}_{k}} \mathbb{E}\left[u_{k}\left(\boldsymbol{p}_{k}, \boldsymbol{p}_{-k}\right) \mid \boldsymbol{h}_{k}\right]
$$

where the expectation is taken over the unknown channels $\boldsymbol{h}_{j}=\left(h_{j, j}, h_{i, j}\right)$, for all $j \neq i$.

The authors showed again that although each player have more information (its own channel $h_{i, i}$ and its interfering channel $h_{-i, i}$ ) the NE is again obtained by uniformly spreading the total power over all the available channels. Moreover, the corresponding NE is not Pareto optimal. 


\subsubsection{Coalitional Games}

In economics for instance, rational players (e.g., companies or manufacturers) tend to create coalitions to maximize their individual or common benefits. This kind of games is called coalitional games $[17,31]$. In this scenario, a set of actions $\mathcal{S}_{c}$ is associated to each of the $C$ coalitions or groups $\mathcal{K}_{c}, \forall c \in\{1, \ldots, C\}$. The set of actions of the $k^{\text {th }}$ player, $\forall k \in \mathcal{K}_{c}$ is then $\mathcal{S}_{c}$. Each coalition obtains a benefit called value, denoted $v_{c}$ for the $c^{\text {th }}$ coalition, as a result of the actions of all the players $k \in \mathcal{K}_{c}$. The total benefit of the coalition is shared between all the members of the coalition. Each coalition can have a different policy $u_{c}\left(\mathcal{K}_{c}, v_{c}\right)$ for sharing the common benefit. Hence, the goal of each player $k, \forall k \in \mathcal{K}$ is to choose the best coalition to join. In fact, rational players will join the coalition where the highest individual benefit is obtained.

Definition 1.4.4 (Coalitional Game) A coalitional game is completely described by the following parameters:

- A finite set of $K$ players, $\mathcal{K}=\{1, \ldots, K\}$.

- A finite set of $C$ coalitions $\mathcal{C}=\{1, \ldots, C\}$ such that $\forall k \in \mathcal{C}, \mathcal{K}_{k} \subseteq \mathcal{K}$ and $\forall i \neq j$, $\mathcal{K}_{i} \cap \mathcal{K}_{j}=\{\}$.

- $C$ finite sets of actions $\mathcal{S}_{1}, \ldots, \mathcal{S}_{C}$, with $\mathcal{S}=\mathcal{S}_{1} \times \ldots \times \mathcal{S}_{C}$.

- A finite set of $C$ values, $\left\{v_{c}: \mathcal{K} \times \mathcal{S} \rightarrow \mathbb{R}: \forall c \in \mathcal{C}\right\}$.

- A finite set of $C$ policies, $u_{c}\left(v_{c}\right), \forall c \in \mathcal{C}$.

A stable outcome or equilibrium of coalitional games consists of a distribution of the players among the different coalitions where no player is interested in choosing another coalition. A common equilibrium is called the grand coalition. Here, there is only one coalition and all the players belong to it. Thus, none of the players can obtain a higher benefit by leaving the grand coalition. Coalitional games are of high importance in the DSA problem. In the OSA model for example, terminals of the same physical layer technology i.e., which can "understand" each other, can ally in order to improve its individual or common performance. 
Similarly, radio devices with similar power constraints or even QoS requirements might be interested in forming/joining a coalition. Nonetheless, this assumption requires radio devices to coordinate themselves in order to establish the available set of actions for a possible coalition. Often, coordination requires signaling among the terminals, which is not always practically appealing.

In the literature, the DSA problem has been modeled as a coalitional game in $[21,22,23]$. The idea of a coalition in the DSA problem for a unique channel $N=1$, and a finite number of radio devices, is that receivers which belong to the same coalition jointly decode their received signals and perform interference cancelation [38]. Indeed, all the other signals from the transmitter belonging to other coalitions are treated as an AWGN. This configuration corresponds to a set of single input multiple output - multiple access channels (SIMO-MAC). Similarly, transmitters can also form coalitions, which will lead us to virtual MISO systems. If both transmitters and receivers form coalitions the network is equivalent to a set of virtual MIMO channels.

The value $v_{c}, \forall c \in\{1, \ldots, C\}$ in both cases, transmitter or receiver cooperation, is then chosen as the sum of individual data rates $R_{k}$ achieved by all the players $k \in \mathcal{K}_{c}$, i.e.,

$$
v_{k}=\sum_{i \in \mathcal{K}_{k}} R_{i}
$$

Depending on its achieved individual data rate $R_{k}$, each player $k$ decides which coalition to joint. For instance, in the receiver cooperation case, depending on the coalition a receiver belongs to, it will decode a different set of interferers. The authors in [21] showed that the grand coalition maximizes the spectrum utilization. This is equivalent to the fact that terminals should jointly decode all the surrounding terminals' signals [38].

\subsection{Hierarchical Spectrum Access Games}

The hierarchical spectrum access model considers two different approaches, the underlaying and the overlaying approaches $[3,4]$. On the one hand the opportunistic devices have to 
meet a certain power constraint in order to keep the interference level they generate on the primary systems always below their noise floor. This is the case of ultra wide band or interference alignment systems for example [4, 44, 45]. Here, the opportunistic and primary transmitters can simultaneously transmit without generating harmful interference on the primary receivers.

On the other hand, the overlaying approach which targets the spectral white spaces i.e., spectrum access opportunities, does not impose any limit on the transmit power. It only requires opportunistic radio devices to identify radio resources left unused by the primary network and exploit them subject to the constraint that it can be required by the primary system at any time. In this approach, the opportunistic and primary players do not transmit simultaneously. If the current power constraints and the available channels are perfectly identified, in both the overlaying and underlaying models, the influence on the primary systems can be neglected and the problem can be modeled as in Sec. 1.2. Hence, all the game theoretical machinery we have developed so far directly applies to this particular dynamic spectrum access scheme.

A special case of hierarchical spectrum access arises when interaction between the primary system and the opportunistic devices is allowed. Here, any action of the primary devices affects the benefit obtained by the opportunistic radio devices. For example, a primary system might offer a set of channels to the opportunistic terminals in exchange of cooperation in the form of distributed space-time coding (DSTC) [16]. This kind of interactions cannot be modeled with the tools presented above. Here, there are two different types of players (primary and secondary) whose priority is different when they access the radio resources. The framework provided by game theory to study this interaction is called Stackelberg games.

\subsubsection{Stackelberg Games}

Here we investigate situations where there is a hierarchy between players. A useful case of this kind of games are Stackelberg games, which were initially introduced by Stackelberg in 1934 [17]. In this game there is an implicit concept of hierarchy upon the set of players. 
Such hierarchy naturally occurs when users are playing sequentially. For example, in a 2level Stackelberg game, the game leader moves first and the other players follow and play simultaneously. The game leader perfectly knows the set of strategies and the utilities of the followers. Similarly, it is guaranteed that the followers can observe the actions of their leader(s).

Definition 1.5.1 (Stackelberg Games) A Stackelberg game is a two-stage game at which one player (leader) moves at the first stage and all the other players (followers) react simultaneously at the second stage.

A Stackelberg game can be easily solved through the concept of sub-game perfect Nash equilibrium. In the first stage the leader, which perfectly knows all the followers set of actions and utility functions, chooses the action that maximizes its benefit considering that each follower will react with the action which maximizes its own benefit as well. Thus, the game leader analyzes all the possible outcomes and picks up the action which maximizes its benefit considering the optimal moves for each player. In the recent literature, an interesting application of Stackelberg games in dynamic spectrum access was presented by Simeone et al.. The problem is modeled as follows. There exists a unidirectional primary link from transmitter $T x_{P}$ to receiver $R x_{P}$ and an ad hoc network with a set $\mathcal{J}$ of $K$ point-to-point links as described in Sec. 1.2. The primary system divides its transmission ( $L$ bit durations) into two parts of $\alpha L$ bit durations and $(1-\alpha) L$ bit durations, with $0 \leqslant \alpha \leqslant 1$. The first $(1-\alpha) L$ bits are dedicated to a direct transmission from $T x_{P}$ to $R x_{P}$. The second $\alpha L$ bits are again divided in two. One part $\beta \alpha L$,with $0 \leqslant \beta \leqslant 1$, is dedicated to send information from $T x_{P}$ to $R x_{P}$ using the ad hoc network as a mean to perform distributed space time coding (DSTC). The remaining $\alpha(1-\beta) L$ bits are then granted to the ad hoc network to transmit its own data. The performance of the ad hoc network follows the one described in Sec. 1.2. The aim of the primary link is described depending on its available information. For the case where instantaneous CSI is available i.e., the primary system perfectly knows all the channel gains, its utility function, $u_{\text {leader }}$, is described in terms of the achieved data rate. A complete discussion is presented in [16]. If only the statistics of the channel realizations are available the utility function is described in terms of the outage probability. In both 
cases, the goal of the leader is to maximize its own utility by deciding on the amount of bits to be coded using DSTC, the amount of bits to grant to the ad hoc network, i.e., $\alpha$ and $\beta$, and the most convenient set of links to use in the DSTC, denoted $\mathcal{K}_{s} \subseteq \mathcal{K}$. Hence, the set of actions of the leader is

$$
\mathcal{S}_{\text {leader }}=\left\{\alpha, \beta, \mathcal{K}_{s}: 0 \leqslant \alpha \leqslant 1,0 \leqslant \beta \leqslant 1, \mathcal{K}_{s} \subseteq \mathcal{K}\right\}
$$

The primary network is considered to be the leader of the game and decides the optimal parameters $\alpha^{*}, \beta^{*}$ and $\mathcal{K}_{s}^{*}$ at the first instant of the game. The optimal values then, correspond to the solution to

$$
\max _{\alpha, \beta, \mathcal{K}_{s}} u_{\text {leader }}\left(\alpha, \beta, \mathcal{K}_{s}\right) \text { s.t. } \mathcal{K}_{s} \subseteq \mathcal{K}, 0 \leqslant \alpha, \beta \leqslant 1
$$

Later, the ad hoc network reacts by exploiting the $\alpha(1-\beta) L$ bit periods it has to transmit. Each transmitter in the ad hoc network plays the game described in Sec. 1.2. The primary player always obtains the best outcome from the game since it is privileged to play at the first moment. The followers, which follow the configuration discussed in Sec. 1.2 obtain a stable non-Pareto NE outcome. This NE has been already studied in Sec. 1.4.

Other Stackelberg formulation is presented in $[56,57]$ for the case of MAC with $N=1$ and the energy-efficient utility function (Eq. 1.10). Therein, the interactions among the transmitters are modeled as in Def. 1.3.1 and a certain degree of hierarchy is introduced in two different ways: 1. Assuming single-user decoding at the receiver, one player (e.g., one primary transmitter) plays first whereas the other players (e.g., secondary transmitters) are assumed to react to the leader's decisions; 2. Assuming neither leader nor followers among the players, hierarchy is introduced by assuming successive interference cancelation at the receiver. It is shown that these two hierarchical models not only improve the individual energy efficiency of all the users but can also be a way of ensuring the existence of an equilibrium and reaching a desired trade-off between the global network performance at the equilibrium and the requested amount of signaling.

In the first case, the authors consider without loss of generality (but possibly with loss of optimality) that user $i$ is the leader of the game (and plays first). Each follower $j \neq$ 
$i$ therefore plays a non-cooperative game with the other followers, given what the leader plays. Interestingly, it is possible to show that, under realistic conditions, there is a unique equilibrium in this hierarchical game, which is called a Stackelberg Equilibrium (SE). To define an SE, let $\boldsymbol{U}^{*}\left(p_{i}\right)$ be the set of NE for the group of followers when the leader plays strategy $p_{i}$. In other words, the leader maximizes its utility function which depends on the NE $u^{*} \in \boldsymbol{U}^{*}\left(p_{i}\right)$ of the followers. By denoting $\left(p_{i}^{\mathrm{SE}}, \boldsymbol{p}_{-i}^{\mathrm{SE}}\right)$ the power profile at the SE, this definition translates mathematically by

$$
p_{i}^{\mathrm{SE}}=\arg \max _{p_{i}} u_{i}\left(p_{1}^{\mathrm{SE}}\left(p_{i}\right), \ldots, p_{i-1}^{\mathrm{SE}}\left(p_{i}\right), p_{i}, p_{i+1}^{\mathrm{SE}}\left(p_{i}\right), \ldots, p_{K}^{\mathrm{SE}}\left(p_{i}\right)\right),
$$

where $p_{j}^{\mathrm{SE}}\left(p_{i}\right), j \neq i$, is the power at the NE of the follower $j$ (which depends on $p_{i}$, the leader's action).

Under this formulation the existence and uniqueness of the SE is always ensured [57]. When player $i$ is assumed to be the leader, its optimal power allocation is

$$
p_{i}^{\mathrm{SE}}=\frac{\sigma^{2}}{\left|h_{i}\right|^{2}} \frac{\gamma^{*}\left(1+\beta^{*}\right)}{1-(K-1) \gamma^{*} \beta^{*}-(K-2) \beta^{*}}
$$

and for each follower $j \neq i$,

$$
p_{j}^{\mathrm{SE}}=\frac{\sigma^{2}}{\left|h_{j}\right|^{2}} \frac{\beta^{*}\left(1+\gamma^{*}\right)}{1-(K-1) \gamma^{*} \beta^{*}-(K-2) \beta^{*}},
$$

if the following (sufficient) conditions hold: $\frac{f^{\prime \prime}(0)}{f^{\prime}(0)} \geq 2 \frac{(K-1) \beta^{*}}{1-(K-2) \beta^{*}}$ and

$$
\phi(x)=x\left[1-\frac{(K-1) \beta^{*}}{1-(K-2) \beta^{*}} x\right] f^{\prime}(x)-f(x)
$$

has a single stationary point in $] 0, \gamma^{*}\left[\right.$, where $\gamma^{*}$ is the positive solution of the equation $\phi(x)=0$ and $\beta^{*}$ is the solution to (Eq. 1.14).

In the second case, the receiver is assumed to implement successive interference cancelation (SIC). Under the assumption that players are perfectly decoded, the optimal power 
allocation at the NE is

$$
\forall i \in\{1, \ldots, K\}, p_{i}^{\mathrm{SIC}}=\frac{\sigma^{2}}{\left|h_{i}\right|^{2}} \beta^{*}\left(1+\beta^{*}\right)^{i-1}
$$

where player denoted by $i$ is decoded with rank $K-i+1$ in the successive decoding procedure at the receiver. In [57], it is shown that this NE always exists and is unique.

In order to compare both approaches, SUD and SIC, assume a random CDMA system with $R_{i}=100 \mathrm{kbps}$ for all $i \in\{1, \ldots, K\}$. An efficiency-function $f(x)=\left(1-e^{-x}\right)^{M}$ with $M \in\{2,5,10,20,50,100\}$. The corresponding values for $\gamma^{*}(M)$ are respectively $1.25,2.66$,

$3.61,4.51,5.65,6.47$. Fig. 1.2 represents the quantity $\frac{w^{\mathrm{SIC}}}{w^{\mathrm{SUD}}}-1$ in percentage as a function of the spectral efficiency $\alpha=\frac{K}{S}$ for $\operatorname{SNR}[\mathrm{dB}]=6$ and random decoding order. Here, $w^{\text {SIC }}$ and $w^{\mathrm{SUD}}$ represent the obtained utility function in the game when SIC is implemented and the game when leader and followers exist, respectively. The asymptotes $\alpha_{\max }=\frac{1}{\beta^{*}(M)}+\frac{1}{S}$ are indicated by (red) dotted lines and $S$ is the spreading length. The gains are particularly significant when the system load is relatively high i.e., when there is a significant amount of interference to be removed after despreading. In fact, when $\frac{K-1}{S} \gamma^{*} \rightarrow 1^{+}$the non-cooperative game becomes dramatically inefficient since the transmit powers at the equilibrium diverge; here, once again we recall that we assume a non-trivial NE at which the users do not exploit all their power. Otherwise, a user who would saturate his power constraint would maximize his utility by transmitting at $P_{i}^{\max }$.

\subsection{Concluding Remarks}

We have seen that game theory is a natural paradigm to study a network where terminals are competing with each other for a common resource namely the spectrum. We have essentially distinguished between two kind of games: 1. The open spectrum access game where all the transmitter-receiver pairs are equal; 2 . The hierarchical spectrum access game where some transmitters are leaders of the game (primary transmitters) or follower (secondary transmitters). Depending on the context each of these games can be non-cooperative or cooperative. In the first case, the network is totally decentralized and the terminals self- 


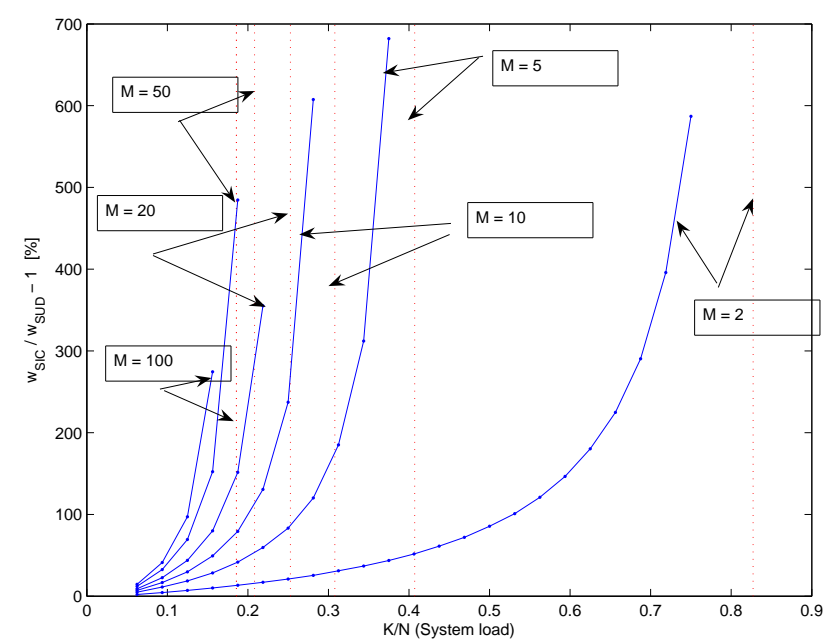

Figure 1.2: Influence of the decoding scheme (SIC/SUD) for different system loads and packet lengths.

ishly optimize their individual performance criteria, which can lead to an equilibrium of the network. The existence and uniqueness of a Nash equilibrium is an attractive feature of a decentralized network since the system owner(s) can predict the performance of the users and therefore guarantee for example a minimum QoS. In the case of cooperative games, the global performance of the network becomes the main target. We have presented several ways of introducing cooperation in a game: repeating the game, forming coalition or by introducing certain degree of hierarchy. The global network efficiency can be measured in terms of price of anarchy or by considering Pareto optimality. Another way to measure it when transmission rates are considered for the users' utilities is the ratio of the network sum-rate at the equilibrium to that obtained by the equivalent virtual MIMO system. Clearly, the assumed spectrum sensing technique will play an important role in selecting the way of cooperating. Also, depending on the a priori information available to the transmitters or/and the used spectrum sensing technique, the game can be with or without complete information. In this case we have seen that the Bayesian approach is a possible candidate to deal with this kind of (practical) situations.

We will conclude this chapter by mentioning a few open issues related to the open spectrum access problem. It is a fact that there are more and more devices using unlicensed bands. Each actor e.g., a telecom operator or an manufacturer generally designs its system independently of the other actors. But this assumption is likely to become less and less valid 
as the number of devices operating in these bands grows. The problem of system interference naturally appears, which creates interaction between the different transmitter-receiver pairs. Obviously there is going to be a game between many players (operators, manufacturers, etc.) who will have to design their terminals in a smart way to take into account this interaction. The problem is particularly challenging since networks will be by nature heterogeneous. Many questions arise. Will the different players continue to deploy their systems independently or will them try to cooperate to mitigate the interference? What kind of information can be reasonable to be assumed at a given terminal in a context where the system owners do not disclose their strategies? Is it possible to classify the different devices in order to apply a Bayesian approach that is robust to the uncertainty on the environment? This chapter gathers a few basic elements released in the recent literature on spectrum access games to start solving these issues but more and more contributions will be needed to provide reliable answers. 


\section{References}

[1] US FCC Spectrum Policy Task Force. "Report of the spectrum efficiency working group". Tech. Rep., November 2002.

[2] S. Haykin, "Cognitive radio: brain-empowered wireless communications". IEEE Journal on Selected Areas in Communications, vol. 23, no. 2, pp. 201-220, 2005.

[3] M. Buddhikot, "Understanding dynamic spectrum access: Models, taxonomy and challenges". IEEE DySPAN, April 2007.

[4] Q. Zhao and B. M. Sadler, "A survey of dynamic spectrum access". IEEE Signal Processing Magazine. vol. 24, no. 3, pp. 79-89, 2007.

[5] P. Leaves, K. Moessner, R. Tafazolli, D. Grandblaise, D. Bourse, R. Tonjes, and M. Breveglieri, "Dynamic spectrum allocation in composite reconfigurable wireless networks". IEEE Communications Magazine, vol. 42, no. 5, pp. 72-81, 2004.

[6] European Radiocommunications Committee, "Relating to the use of short range devices, ERC Recommendation 70-03”. Apr. 2002.

[7] A. Mackenzie, L. DaSilva, "Game Theory for Wireless Engineers (Synthesis Lectures on Communications)". 1st ed. Morgan \& Claypool Publishers, May 2006.

[8] L. Berlemann, G. R. Hiertz, B. Walke, and S. Mangold, "Strategies for distributed QoS support in radio spectrum sharing". IEEE International Conference on Communications, ICC 2005, vol. 5, pp. 3271-3277, 2005.

[9] S. Shakkottai, E. Altman, and A. Kumar, "Multihoming of users to access points in WLANS: A population game perspective". IEEE Journal on Selected Areas in Communications, vol. 25, no. 6, pp. 1207-1215, 2007.

[10] P. Nurmi, "Modelling routing in wireless ad hoc networks with dynamic Bayesian games". First Annual IEEE Communications Society Conference on Sensor and Ad Hoc Communications and Networks, pp. 63-70, 2004.

[11] A. B. Mackenzie and S. B. Wicker, "Game theory and the design of self-configuring, adaptive wireless networks". IEEE Communications Magazine, vol. 39, no. 11, pp. 126$131,2001$.

[12] V. Srivastava, J. Neel, A. B. Mackenzie, R. Menon, L. A. Dasilva, J. E. Hicks, J. H. Reed, and R. P. Gilles, "Using game theory to analyze wireless ad hoc networks". IEEE Communications Surveys \& Tutorials, vol. 7, no. 4, pp. 46-56, 2005. 
[13] S. M. Perlaza, L. Cottatellucci, and M. Debbah, "A game theoretic framework for decentralized power allocation in IDMA systems". IEEE International Symposium on Personal, Indoor and Mobile Radio Communications, September 2008.

[14] N. Bonneau, M. Debbah, E. Altman, and Are Hjorungnes, "Non-atomic games for multi-user systems". IEEE Journal on Selected Areas in Communications, issue on Game Theory in Communication Systems, 2008.

[15] F. Meshkati, M. Chiang, H. V. Poor, and S. C. Schwartz, "A game-theoretic approach to energy-efficient power control in multicarrier CDMA systems". IEEE Journal on Selected Areas in Communications, vol. 24, no. 6, pp. 1115-1129, 2006.

[16] O. Simeone, I. Stanojev, S. Savazzi, Y. Bar-Ness, U. Spagnolini, and R. Pickholtz, "Spectrum leasing to cooperating secondary ad hoc networks". IEEE Journal on Selected Areas in Communications, vol. 26, no. 1, pp. 203-213, 2008.

[17] D. Fudenberg and J. Tirole, "Game Theory". MIT Press, October 1991.

[18] A. Carleial, "Interference channels". IEEE Transactions on Information Theory, vol. 24, no. 1 , pp. 60-70, 1978.

[19] H. Sato, "Two-user communication channels". IEEE Transactions on Information Theory, vol. 23, no. 3, pp. 295-304, 1977.

[20] R. J. Aumann, "Subjectivity and correlation in randomized strategies". Journal of Mathematical Economics, vol. 1, no. 1, pp. 67-96, March 1974.

[21] S. Mathur, L. Sankaranarayanan, and N. B. Mandayam, "Coalitional games in Gaussian interference channels". IEEE International Symposium on Information Theory, 2006, pp. 2210-2214.

[22] ——, "Coalitional games in receiver cooperation for spectrum sharing". 40th Annual Conference on Information Sciences and Systems, pp. 949-954, 2006.

[23] — - "Coalitional games in cooperative radio networks". Fortieth Asilomar Conference on Signals, Systems and Computers, pp. 1927-1931, 2006.

[24] L. J. Savage, "Foundations of Statistics". 1st ed. John Wiley, 1954.

[25] R. G. Wendorf and H. B. Seidenberg, "Channel-change games for highly interfering spectrum-agile wireless networks".2nd International Symposium on Wireless Pervasive Computing, 2007.

[26] R. G. Wendorf and H. Blum, "A channel-change game for multiple interfering cognitive 
wireless networks". Military Communications Conference, pp. 1-7, 2006.

[27] — - "Wlc38-3: Simple channel-change games for spectrum agile wireless networks". IEEE Global Telecommunications Conference, 2006, pp. 1-5.

[28] S. Mangold, L. Berlemann, and B. Walke, "Equilibrium analysis of coexisting IEEE 802.11e wireless LANs". 14th IEEE Symposium on Personal, Indoor and Mobile Radio Communications, 2003.

[29] — - "Radio resource sharing model for coexisting IEEE 802.11e wireless LANs". International Conference on Communication Technology, vol. 2, pp. 1322-1327, 2003.

[30] L. Berlemann, G. R. Hiertz, B. H. Walke, and S. Mangold, "Radio resource sharing games: enabling QoS support in unlicensed bands". IEEE Network, vol. 19, no. 4, pp. 59-65, 2005.

[31] M. J. Osborne, "An Introduction to Game Theory". Oxford University Press, USA, August 2003.

[32] F. Willems, "The discrete memoryless multiple access channel with partially cooperating encoders (corresp.)". IEEE Transactions on Information Theory, vol. 29, no. 3, pp. 441445, 1983.

[33] A. Sendonaris, E. Erkip, and B. Aazhang, "User cooperation diversity. part I. system description". IEEE Transactions on Communications, vol. 51, no. 11, pp. 1927-1938, 2003.

[34] V. Belmega, S. Lasaulce, and M. Debbah, "Decentralized handovers in cellular networks with cognitive terminals". 3rd IEEE International Symposium on Communications, Control and Signal Processing, March 2008.

[35] R. Etkin, A. Parekh, and D. Tse, "Spectrum sharing for unlicensed bands". IEEE Journal on Selected Areas in Communications, vol. 25, no. 3, pp. 517-528, 2007.

[36] R. Etkin, "Spectrum sharing: Fundamental limits, scaling laws, and self-enforcing protocols". Ph.D. dissertation, EECS Department University of California, Berkeley, December 2006.

[37] C. H. Papadimitriou, "Algorithms, games, and the internet". 28th International Colloquium on Automata, Languages and Programming, Springer-Verlag, pp. 1-3, 2001.

[38] S. Verdú, "Multiuser Detection". Cambridge University Press, August 1998.

[39] Cover, Thomas and Thomas, Joy. "Elements of Information Theory". John Wiley and 
Sons, New York, 1991.

[40] S. Boyd and L. Vandenberghe, "Convex Optimization". Cambridge University Press, March 2004.

[41] Z.-Q. Luo and J.-S. Pang, "Analysis of iterative waterfilling algorithm for multiuser power control in digital subscriber lines". EURASIP J. Appl. Signal Process., no. 1, p. $80,2006$.

[42] J. C. Harsanyi, "Games with incomplete information played by Bayesian players, I-III". Manage. Sci., vol. 50, no. 12 Supplement, pp. 1804-1817, 2004.

[43] S. Adlakha, R. Johari, and A. Goldsmith, "Competition in wireless systems via Bayesian interference games". arXiv:0709.0516v1, Sep 2007.

[44] L. S. Cardoso, M. Kobayashi, M. Debbah, and O. Ryan, "Vandermonde frequency division multiplexing for cognitive radio". IEEE Signal Processing Advances in Wireless Communications, July 2008.

[45] S. M. Perlaza, M. Debbah, S. Lasaulce, and J.-M. Chaufray, "Opportunistic interference alignment in MIMO interference channels". IEEE International Symposium on Personal, Indoor and Mobile Radio Communications, September 2008.

[46] R. G. Cascella, "The value of reputation in peer-to-peer networks". 5th IEEE Conference on Consumer Communications and Networking, pp. 516-520, 2008.

[47] Z. Ji and K. J. R. Liu, "Collusion-resistant dynamic spectrum allocation for wireless networks via pricing". 2nd IEEE International Symposium on New Frontiers in Dynamic Spectrum Access Networks, pp. 187-190, 2007.

[48] Meshkati, Farhad, "Game-theoretic Approaches to Energy-efficient Resource Management in Wireless Networks". Ph.D. dissertation. Department of Electrical Engineering, Princeton University. 2006.

[49] Rodriguez, Virgilio, "An Analytical Foundation for Resource Management in Wireless Communications". IEEE Global Telecommunications Conference, San Francisco, CA, 1-5 December 2003.

[50] Yu, Wei and Ginis, G. and Cioffi, J. M, "Distributed multiuser power control for digital subscriber lines". IEEE Journal on Selected Areas in Communications, 2002.

[51] Yu, Wei and Ginis, G. and Cioffi, J. M, "An adaptive multiuser power control algorithm for VDSL". IEEE Global Telecommunications Conference. December 2001. 
[52] Chung, Seong T. and Kim, Seung J. and Lee, Jungwon and Cioffi, J. M. "A gametheoretic approach to power allocation in frequency-selective gaussian interference channels". IEEE International Symposium on Information Theory, 2003.

[53] Nash, John. "Non-Cooperative Games". Annals of Mathematics, vol. 54. No 2, September 1951.

[54] Nash, John. "Equilibrium Points in n-Person Games". Proceedings of the National Academy of Sciences, 1950.

[55] Basar, Tamer and Olsder, Geert J. "Dynamic Noncooperative Game Theory". Soc for Industrial \& Applied Math, 1998.

[56] Y. Hayel, S. Lasaulce, R. El-Azouzi, and M. Debbah, "Introducing Hierarchy in EnergyEfficient Power Control Games". Second International Workshop on Game Theory in Communication Networks, 2008.

[57] Y. Hayel, S. Lasaulce, R. El-Azouzi, and M. Debbah, "Introducing Hierarchy in Energy Games". Submitted to IEEE Transactions on Wireless Communications, 2008.

[58] Meshkati, F. and Poor, H. V. and Schwartz, S. C. and Mandayam, N. B, "An energyefficient approach to power control and receiver design in wireless data networks". IEEE Transactions on Communications, pp. 1885-1894, 2005.

[59] Buzzi, Stefano and Poor, Vincent H, "Joint Receiver and Transmitter Optimization for Energy-Efficient CDMA Communications". IEEE Journal on Selected Areas in Communications, vol. 2, pp. 459-472, 2008. 